\title{
PARTICLE MOVEMENT ON A PERFORATED PLATE DISTRIBUTOR OF FLUIDIZED BED
}

\author{
MasayUKi HORIO, Hiroshi KIYOTA and IwaO MUCHI \\ Department of Iron and Steel Engineering, \\ Nagoya University, Nagoya 464
}

\begin{abstract}
Particle motion in the grid zone above a perforated plate distributor was investigated with 15-cm $\phi$ circular and 10-cm $\phi$ semicircular fluidized beds. Tracer method was applied to observe dead zone shape and particle movement visually. The particle motion in the grid zone was found to be rather stable, similarly to that in a spouted bed. Particle entrainment rate in a jet was determined from the observed values of particle stream function in the annular region. This particle circulation, which is the essential scheme of gas-solid contact in grid zone, is mainly controlled by the gas velocity through an orifice. Dead zone is formed under a mechanism similar to that of bulk solids flow in a bin. Correlations are presented for estimating the size of true and pseudo-dead zones and the particle turnover rate in the pseudo-dead zone.
\end{abstract}

\section{Introduction}

Distributor design is one of the most significant items in fluidized-bed design and a number of criteria ${ }^{4,5,7,8,20,16,18,19,24)}$ have been proposed in regard to the quality of fluidization, such as even distribution of gas and desirable bubble size. However, there seems to be almost no criterion which can take into account the phenomena in the grid zone. The importance of gas and solid contact in this zone for the case of rapid reactions was pointed out by Zenz ${ }^{24}$ and analysed by Behie $e t a l .^{2)}$ and Mori and Wen ${ }^{17)}$.

The characteristics of gas jets on a perforatedplate distributor has been investigated by many authors and several correlations are available for maximum jet height $t^{1,15,21)}$. Particle motion in the grid zone has been another topic of interest. Formation of the dead zone has been studied recently ${ }^{3,6,12,13,20-23)}$, but sufficient information has not been established because of the complex geometry of the dead zone and of the difficulty in three-dimensional measurements.

To evaluate the effect of the grid zone on the conversion of fluidized bed reactions, it is necessary to know both gas and solid motions. There exists a similarity between gas and particle motions around a jet and those in a spouted bed, as already mentioned by Lefroy and Davidson". Fluid dynamic models of spouted beds have been proposed by Mamuro and Hattori $^{14)}$, Lefroy and Davidson ${ }^{91}$ and $\operatorname{Lim}$ and Mathur ${ }^{11}$, but complete solutions both for gas and solid phases have not been presented to date.

It is the objective of this paper to establish the

Received May 14, 1979. Correspondence concerning this article should be addressed to M. Horio. mechanistic understanding of particle motion above a perforated-plate distributor. The distribution of dead zone and the particle flow pattern close to jets are investigated by direct visual measurements in circular and semicircular fluidized beds.

\section{Experiment}

\section{1 Experimental apparatus and particles}

A fluidized-bed column of $15 \mathrm{~cm} \mathrm{I.D.} \mathrm{and} 106 \mathrm{~cm}$ height (Bed A) and a semicircular fluidized-bed column of $10 \mathrm{~cm} \mathrm{I}$. D. and $50 \mathrm{~cm}$ height (Bed B) were used for the present investigation.

Bed A was made of an iron tube and a nichrome wire heater was fitted on the outer surface of the column to heat the bed for the wax (paraffin) fixation experiment. The distributors for Bed A were 1 to $2 \mathrm{~mm}$ thick perforated aluminium plates. Four different distributors were used, and their specifications are listed in Table 1. To prevent the falling back of particles, 200-mesh wire gauze was attached to the lower surface of the perforated plate. Entrained particles were collected by a cyclone and continuously fed back to the bed surface.

Bed B was made of PVC tube. The flat vertical wall and the distributor plate at the bottom were made

\begin{tabular}{|c|c|c|c|c|c|}
\hline \multicolumn{6}{|c|}{ Table 1 Distributors } \\
\hline \multirow{2}{*}{$\begin{array}{l}\text { Bed } \\
\text { No. }\end{array}$} & \multirow[b]{2}{*}{ I } & \multicolumn{3}{|c|}{ A $(15 \operatorname{cm} \phi$, circular $)$} & \multirow{2}{*}{$\begin{array}{c}\mathrm{B}(10 \mathrm{~cm} \phi \\
\text { semicircular }) \\
\mathrm{V}\end{array}$} \\
\hline & & II & III & IV & \\
\hline$P_{n}[\mathrm{~cm}]$ & 2 & 3 & 4 & 4 & 4 \\
\hline$d_{n}[\mathrm{~mm}]$ & 1.5 & 2 & 3 & 4 & 3 \\
\hline Number of & & & & & \\
\hline orifices & 48 & 21 & 12 & 12 & 3 \\
\hline Opening ratio & 0.0048 & 0.0037 & 0.0048 & 0.0086 & 0.0036 \\
\hline
\end{tabular}


Table 2 Particles used in the experiments

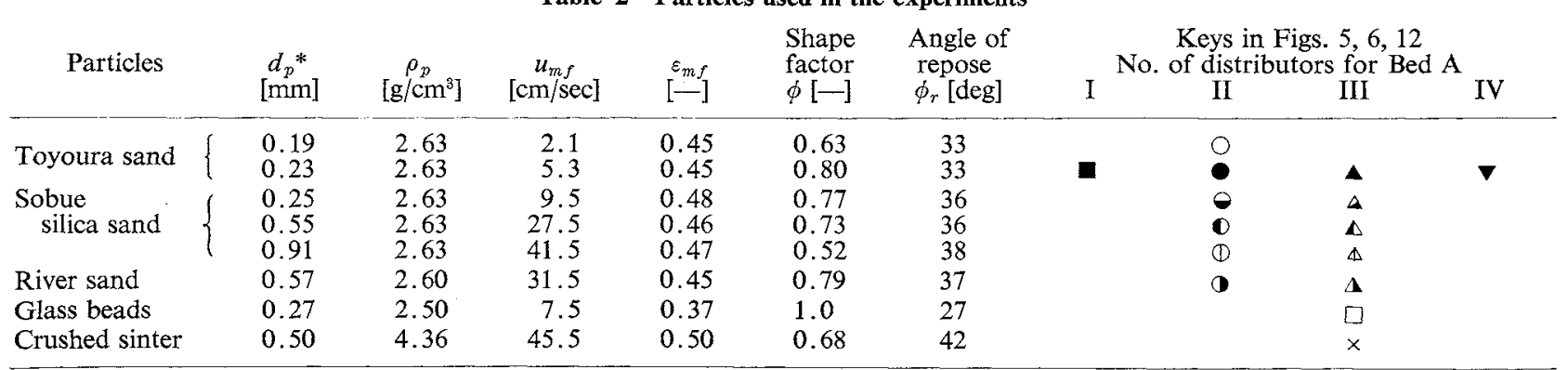

* surface volume mean diameter
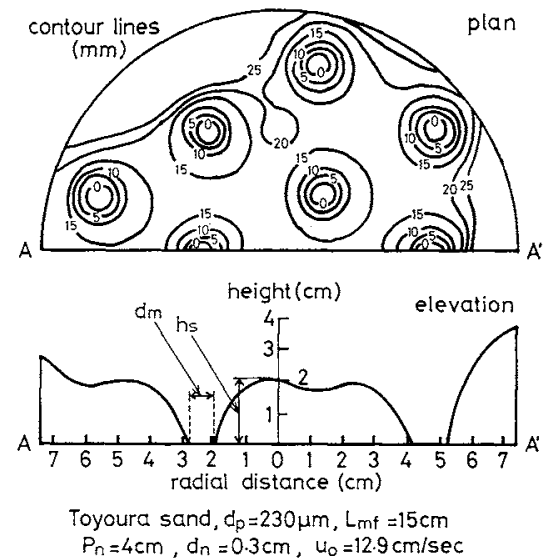

Fig. 1 Example of dead-zone boundary on a perforated plate distributor

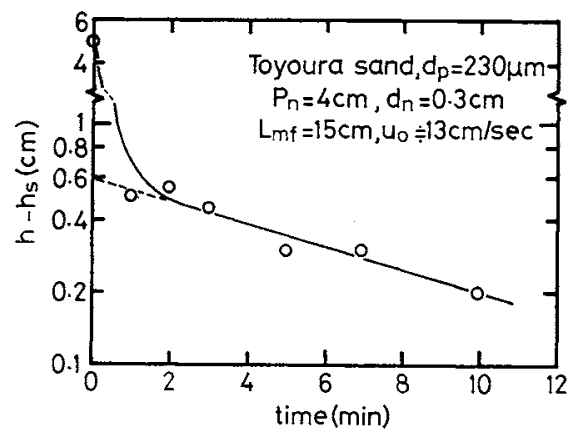

Fig. 2 Effect of duration of fluidization on height of tracer zone

of 5-mm thick transparent PVC plates so that the particle motion around a jet could be visually observed. A copper tube of $3 \mathrm{~mm} \mathrm{I}$. D. was attached to each orifice for air supply. The number and pitch of orifices can be found in Table 1. The top of the tube was covered by wire gauze and its level was adjusted to be equal to that of the upper surface of the distributor plate. The cross-section of the two orifices on the front was made into a semicircular shape. The flow rate to each orifice was controlled so that the observed jet height for every orifice became equal in a shallow fluidized bed.

Table 2 shows the properties of particles. Some portions of the particles were dyed by ink for tracer use but no significant difference in the physical properties was caused by dyeing.

\section{2 Experimental method}

To observe the dead zone pattern visually in a three-dimensional bed, the wax fixation technique was applied. Prior to fluidization, tracer particles were charged up to about $5 \mathrm{~cm}$ from the distributor plate and over this layer ordinary particles were charged. The bed height at incipient fluidization was $15 \mathrm{~cm}$ in all cases. After the bed was fluidized for a certain period at a constant flow rate, the supply of air was stopped. The bed was heated to assure complete permeation of hot paraffin. The bed surface was covered with a paper filter to prevent the movement of particles while it was irrigated. Then the bed was allowed to cool. The bed fixed with paraffin was taken out of the column and sliced to determine the dead zone shape.

In the semicircular bed (Bed B) the motion of tracer particles mixed into the particle moving zone was observed from the front wall visually and by photographs.

\section{Results from Bed $A$}

\section{1 Shape of particle dead zone}

A typical example of the observed boundary between the tracer zone and the fully mixed zone after five minutes fluidization is shown in Fig. 1. The tracer zone is understood to represent the particle dead zone approximately. It can be found that particles are actively moving in the horn shaped region above each orifice.

The volume of the tracer zone gradually decreases with time and approaches to the true dead zone. Figure 2 shows the change of maximum height of the tracer zone, $h$, with time. To get the figure the wax fixation runs were repeated by changing the duration of fluidization. The true dead-zone height, $h_{s}$, was approximated by the height of the tracer zone after 60 minutes fluidization. From Fig. 2 it is found that the difference $h-h_{s}$ changes exponentially with time. The intersection of the straight line with the $h-h_{s}$ axis 
gives the thickness of the pseudo-dead zone, i.e. a zone where particles move but much more slowly than in the bulk of the fluidized bed. After a few minutes fluidization the change in tracer-zone height usually becomes negligibly small. In the succeeding runs the duration of fluidization was fixed at five minutes. Since the reducing rate of $h-h_{\mathrm{s}}$ is slower for lower gas velocity, as observed in a two-dimensional bed previously ${ }^{19}$, there is a certain possibility that the observed tracer zone still includes some part of the pseudo-dead zone when gas velocity is low. However, in most cases five minutes fluidization is expected to be sufficient to give a tracer zone height sufficiently close to $h_{s}$.

\subsection{Effect of orifice pitch and gas velocity on dead- zone shape}

The major factors affecting dead-zone shape are center-to-center orifice pitch, $P_{n}$, and the gas velocity through an orifice, $u_{n}$. The type of particles significantly influences the dead-zone shape, as shown later.

The linear relationship between $h_{s}$ and $P_{n}$ is shown in

Fig. 3. Figure 4 shows the effect of gas velocity on the dead-zone shape. As illustrated in Fig. 1, let us define $d_{m}$ as the diameter of the particle moving zone on the distributor plate. In the high gas velocity range, where the observed tracer zone is supposed to represent the true dead zone, $d_{m}$ increases proportionally with decrease in $h_{s}$ while the angle of tracer zone edge on the distributor remains constant. Let us call this angle the angle of dead zone. It is important here to note that the angle of dead zone is close to the angle of repose, as can be seen in Fig. 4.

\subsection{Diameter of particle moving zone on distributor}

Figure 5 shows the relationship between $u_{n}$ and $d_{m}$ / $d_{n}$, where the latter is the ratio of particle moving zone diameter to orifice diameter. Orifice pitch is found to have little effect on particle moving zone diameter. The present data can be correlated in the following form:

$$
d_{m} / d_{n}=4.4 \times 10^{-6} u_{n}^{1.5}+1.75 \quad\left[u_{n}: \mathrm{cm} / \mathrm{sec}\right]
$$

and $90 \%$ of the data are within $\pm 35 \%$ of the correlation.

\section{4 Correlation for dead zone height}

In Fig. 6 an example of the vertical cross-section of the dead zone is shown, where point $A$ denotes the dead-zone top and $\overline{\mathrm{BC}}$ equals $\left[(2 / \sqrt{3}) P_{n}-d_{m}\right] / 2$. Point $\mathrm{A}^{\prime}$ is taken so that $\overline{\mathrm{A}^{\prime} \mathrm{C}^{\prime}}$ equals $\overline{\mathrm{AC}}\left(=h_{s}\right)$ and $\angle \mathrm{A}^{\prime} \mathrm{BC}^{\prime}$ equals the angle of the dead zone, which is close to the angle of repose. Segment $\overrightarrow{\mathrm{BC}}^{\prime}$ is close to but less than $\overline{\mathrm{BC}}$. Therefore, $\overline{\mathrm{BC}^{\prime}}$ can be approximated by $\left(P_{n}-d_{m}\right) / 2$, and dead-zone height, $h_{s}$, is expressed by the following equation:

$$
h_{s}=(1 / 2) \cdot\left(P_{n}-d_{m}\right) \tan \phi_{r}
$$

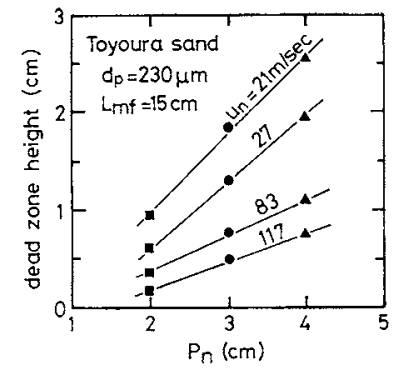

Fig. 3 Effect of orifice pitch on dead-zone height

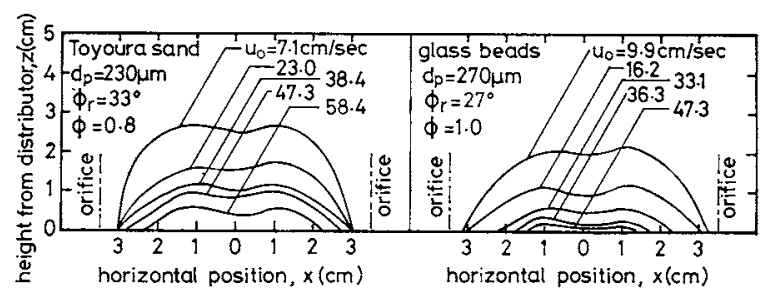

Fig. 4 Comparison of dead-zone shape for particles of different properties

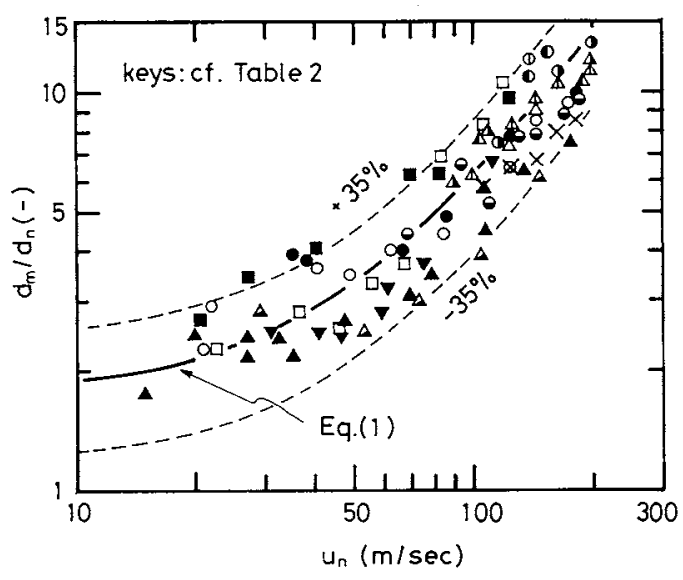

Fig. 5 Moving zone diameter, $\boldsymbol{d}_{m}$, as a function of gas velocity, $\boldsymbol{u}_{n}$, and orifice diameter, $\boldsymbol{d}_{n}$

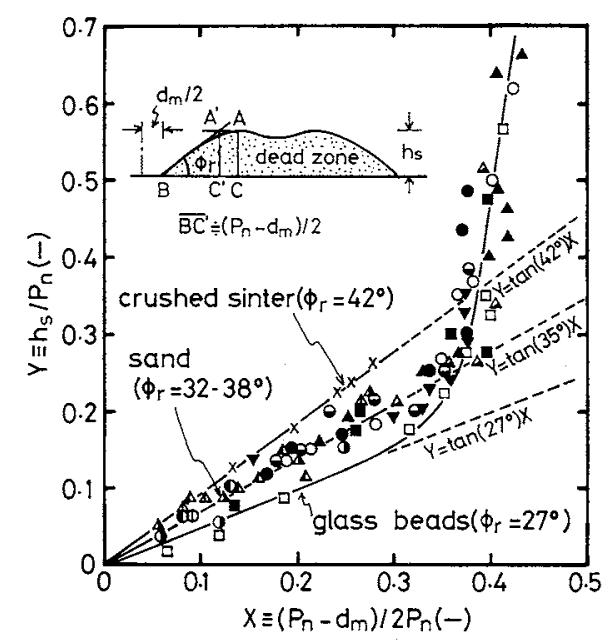

Fig. 6 Correlation of dead-zone height 


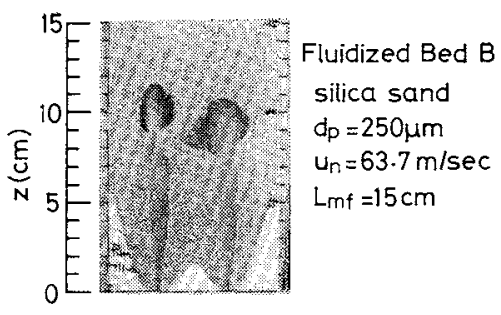

Fig. 7 Example of jetting, dead zone and initial bubble formation

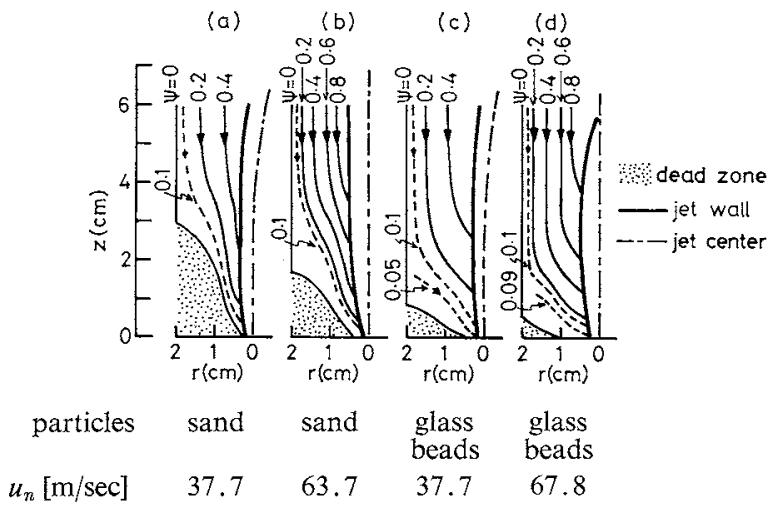

Fig. 8 Observed stream lines around a jet

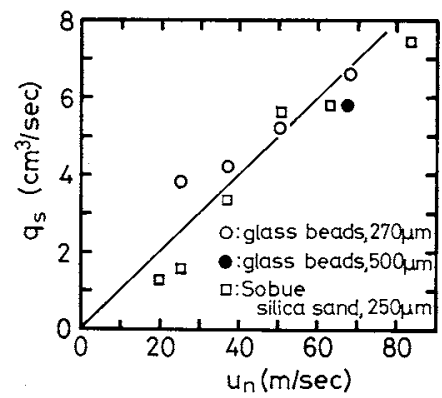

Fig. 9 Relationship between particle circulation rate, $\boldsymbol{q}_{s}$, and orifice gas velocity, $\boldsymbol{u}_{n}$

As shown in Fig. 6 Eq. (2) successfully correlates the result. The existence of data which appear not to satisfy Eq. (2) is only superficial, because these data, which correspond to the lower gas velocity, contain a certain part of the pseudo-dead zone in the observed values of $h_{\mathrm{s}}$ due to the short duration $(5 \mathrm{~min})$ of fluidization. From these data we can determine the particle turnover rate in the pseudo-dead zone, as is discussed later in $\mathbf{4 . 2}$.

\section{Results from Bed B}

\section{1 Flow pattern of particles around a jet}

In a series of experiments using Bed B stable jetting was observed in all runs for beds both of sand and glass beads. An example of jetting and initial bubble formation is shown in Fig. 7.

Figure 8 shows the observed particle stream lines and the values of stream function, $\psi$. The Stokes stream function, $\phi=\oint v_{s} y d s$, was computed so that $\phi$ equals zero at the center of neighbouring orifices. In Fig. 8 $\psi_{i}$, which denotes the value of $\phi$ at the $i$-th stream line, is calculated by Eq. (3),

$$
\phi_{i}=\sum v_{s i} y_{i} \Delta s_{i}
$$

where $v_{s i}$ is the average velocity of particles between $i$-th and $(i+1)$-th stream lines, $y_{i}$ is the distance from jet axis to the middle point of $i$-th and $(i+1)$-th stream lines and $\Delta s_{i}$ is the distance between adjacent stream lines. The measurement of $v_{s}$ is easy in the annular region around the upper part of a jet because of rather slow particle motion in this region. In the present work the location of a tracer particle was dotted on the transparent front wall at constant time intervals so that particle velocity and stream lines were available. Photographs were also used to determine stream lines.

The observed flow pattern of particles is similar to those in the spouted beds reported by Yokogawa ${ }^{2,4}$ and Lim et al. ${ }^{23)}$. The presence of particle moving zone on the distributor plate was confirmed also in Bed B. It is found that there exists a boundary layer over the dead zone. Evidently, this zone is identical to the pseudo-dead zone defined earlier.

It can be seen from Fig. 8 that the angle of approach, $\alpha$, (i. e. the angle of inclination of particle stream lines around a jet measured from the horizontal plane) approximately satisfies the following relationship in the region above the boundary layer for beds both of sand and glass beads:

$$
\alpha=\phi_{r} / 2+\pi / 4
$$

For non-cohesive powder, usually the angle of internal friction. is roughly equal to the angle of repose. Equation (4) thus implies that $\alpha$ is equivalent to the angle of the failure plane derived from the mechanics of plastic fluid. Therefore, the height of the pseudodead zone, $h_{s p}$, can be estimated by Eq. (5).

$$
h_{s p}=(1 / 2) \cdot\left(P_{n}-d_{0}\right) \tan \left(\phi_{r} / 2+\pi / 4\right)
$$

where $d_{m 0}$ is defined as follows:

$$
d_{m 0}=d_{m}\left(u_{n} \fallingdotseq 0\right)
$$

\section{2 Entrainment of solids by a jet}

The total volumetric circulation rate of particles, $q_{s}$, was determined by measuring average particle velocity in the annulus at the level of the jet top. Results are plotted against $u_{n}$ in Fig. 9. The value of $q_{s}$ increases linearly with increase in $u_{n}$, but no influence of the type of particles on $q_{s}$ was evident in the present experiment.

The volumetric flow rate of particles entrained by a jet, $q_{s j}$, is a function of height and can be determined from stream line data such as in Fig. 8. The ratio of $q_{s j}$ to the total particle circulation rate, $q_{s}$, is shown in Fig. 10 for the case of glass beads. It can be found that the absorption of particles into a jet is occurring uniformly in the lower half of total jet height. In the 
case of sand particles the angle of approach was much steeper and the accurate entrance position of each stream line was difficult to locate, but a similar tendency seems to hold.

\section{Discussion}

\section{1 Mechanism of dead-zone formation}

From the present observation the dead-zone boundary does not indicate the region where disturbance by bubble motion can affect particle motion. The major factor of dead-zone formation is understood to be the steady downflow of particles in the region between jets, which seems close to the case of dead-zone formation in a bin. To show this point more clearly, dead-zone formation was studied in a two-dimensional bin with two discharging slits at both sides of the bottom. The bin was $1 \mathrm{~cm}$ thick, $4 \mathrm{~cm}$ wide $\left(=l_{b}\right), 38 \mathrm{~cm}$ high and made of transparent PVC plate. Solids discharge rate was controlled by adjusting the opening of slits. Particles were first drained to obtain a heap showing the angle of repose. The slit was covered by adhesive tape to stop the draining. Then particles were charged to form two layers of different colors. During a discharge run the drained particles were recycled to maintain a constant bed height.

An example of the observed dead zone is shown in Fig. 11, where it is found that the slope of the dead zone is similar to that in fluidized beds and that the angle of the dead zone is almost equal to the angle of repose. To compare the results from the bin with those from Bed B, $2 h_{s} / l_{b}$ and $2 h_{s} /\left(P_{n}-d_{m}\right)$, which approximately indicate the tangent of dead zone angle, are plotted against the average solid down-flow velocity, $v_{s}$, in Fig. 12. As expected, the dead zone height is similar in both cases indicating the identity of the mechanism of dead-zone formation.

\section{2 Particle turnover rate in pseudo-dead zone}

In some situations such as fast exothermic reactions, the distinction between true- and pseudo-dead zones has no practical meaning, unless the particle turnover rate is sufficiently fast in the pseudo-dead zone. By introducing a turnover rate constant $k$ the tracer zone height, $h$, at time $t$ can be expressed by Eq. (7) based on the result of Fig. 2.

$$
\left(h-h_{s}\right) /\left(h_{s p}-h_{s}\right)=\exp (-k t)
$$

From this equation the turnover time for $95 \%$ of the pseudo-dead zone height is given by Eq. (8).

$$
t_{95 \%}=3 / k
$$

Since those data not correlated by the equation $X=X \tan \phi_{r}$ in Fig. 6 are believed to include some amount of the pseudo-dead zone, it is possible to obtain a rough estimation of $k$ from Eqs. (2), (5) and (6) with the observed value of $h$ and the fluidizing period, $t=5 \mathrm{~min}$. In the case of these data $d_{m}$ does

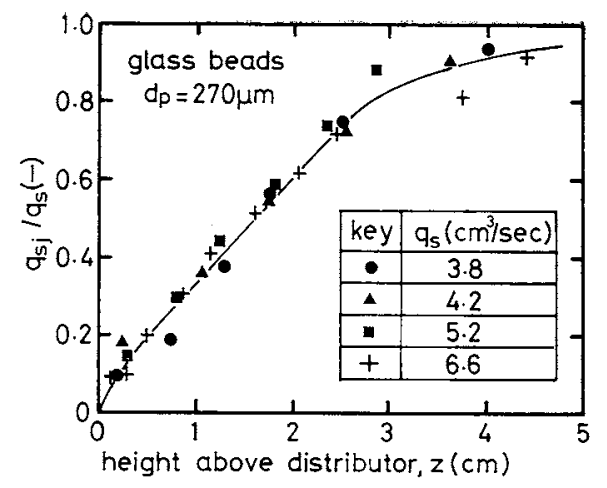

Fig. 10 Fractional entrainment of particles, $\boldsymbol{q}_{s j} / \boldsymbol{q}_{\boldsymbol{s}}$, by a jet

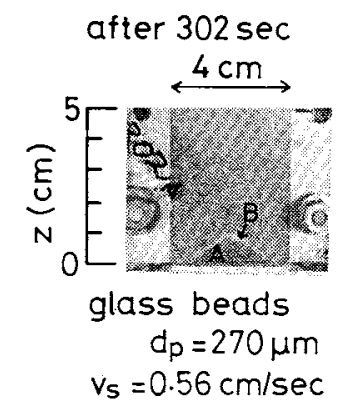

Fig. 11 Two-dimensional bin flow showing a dead zone $(A+B)$ and a heap $A$ for angle of repose measurement

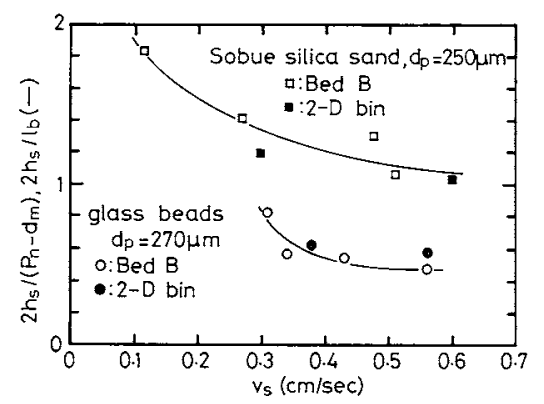

Fig. 12 Comparison of dead-zone heights from Bed $B$ and $2 D$ bin

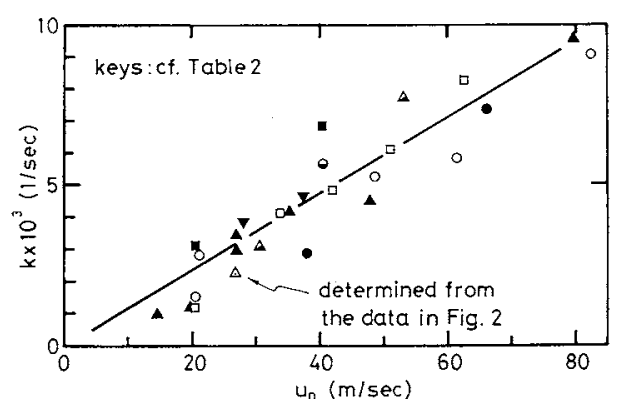

Fig. 13 Relation between particle turnover rate constant, $\boldsymbol{k}$, and orifice gas velocity, $\boldsymbol{u}_{n}$

not change much while $h$ is varying from $h_{s p}$ to $h_{s}$ and the value of $d_{m}$ can be approximated by $d_{m 0}$, which equals $1.75 d_{m}$ from Eq. (1). Results are plotted in Fig. 13 and the data are correlated by Eq. (9). 


$$
k \times 10^{4}=1.2 \times 10^{-2} u_{n}-1 \quad\left[u_{n}: \mathrm{cm} / \mathrm{sec}\right]
$$

\section{3 Gas and solid contact in grid zone}

The gas-solid contacting mechanism in the grid zone is much different from that between bubble and emulsion phases where gas circulation is the rate-controlling step. In the grid zone, particle circulation is the main scheme of gas-solid contact. Behie et al. ${ }^{103}$ proposed the concept of gas-phase mass transfer coefficient between a jet and its surrounding phase and presented a correlation for it. Mori and Wen ${ }^{11)}$ suggested treating the grid zone as a complete mixing cell in reactor modeling. The present results show that the former approach is not realistic. On the other hand, when an important contribution of the grid zone in the final conversion is found by the latter model, it should be concluded that a more realistic evaluation of gas-solid contact is necessary. In such cases solid circulation and the hold-up in a jet would be the major topics of importance.

\section{Conclusion}

Grid zone, i. e. the region below the level of initial bubble formation, has been investigated by threedimensional experimental fluidized beds focusing our attention on particle movement.

It was found that particles in the grid zone are not much influenced by bubble motion, but flow in a stable flow pattern like that in a spouted bed. A wellflowing zone, dead zone and peudo-dead zone have been distinguished. The pseudo-dead zone is the boundary layer between the former two zones. Equations (1), (2) and (5) are presented to estimate the size of these zones.

Entrainment rate of particles in a jet was determined from visual measurements. The absorption of particles by a jet was found to be completed in the lower half of a jet. The particle circulation rate through a jet and the particle turnover rate in the pseudo-dead zone are both proportional to the orifice gas velocity.

The results presented in this paper are to serve as the framework of a systematic description of grid-zone phenomena.

\section{Nomenclature}

$\begin{array}{llr}d_{m} & =\begin{array}{l}\text { diameter of particle moving zone on } \\ \end{array} & \text { distributor } \\ d_{m 0} & \text { value of } d_{m} \text { when gas velocity tends to zero }[\mathrm{cm}] \\ h, h_{s}, h_{s p}= & \text { heights of tracer zone, true and } \\ & \text { pseudo-dead zones } & \\ k & = & \text { particle turnover rate constant in } \\ & \text { pseudo-dead zone } & \\ l_{b} & = & \text { width of two-dimensional bin } \\ P_{n} & = & \text { pitch of orifices }\end{array}$

$$
\begin{aligned}
& q_{s} \quad=\text { total particle circulation rate } \quad\left[\mathrm{cm}^{3} / \mathrm{sec}\right] \\
& q_{s j} \quad=\text { particle flow rate in a jet } \quad\left[\mathrm{cm}^{3} / \mathrm{sec}\right] \\
& u_{n} \quad=\text { gas velocity through an orifice } \quad[\mathrm{cm} / \mathrm{sec}] \\
& \begin{array}{lll}
u_{0} & =\text { superficial gas velocity } \quad[\mathrm{cm} / \mathrm{sec}]
\end{array} \\
& v_{s} \quad=\text { particle velocity } \quad[\mathrm{cm} / \mathrm{sec}] \\
& y \quad=\text { distance from jet axis } \quad[\mathrm{cm}] \\
& \alpha \quad=\text { angle of approach (i. e. angle of particle } \\
& \text { stream line inclination near a jet) [rad] } \\
& \phi_{r} \quad=\text { angle of repose } \quad \text { [rad] }
\end{aligned}
$$

\section{Literature Cited}

1) Basov, V. A., V. I. Markhevka, T. Kh. Melik-Akhnazarov and D. I. Orochko: Int. Chem. Eng., 9, 263 (1969).

2) Behie, L. A. and P. Kehoe: AIChE J., 19, 1070 (1973).

3) Chiba, T. and H. Kobayashi: Preprint of the 12th Autumn Meeting of The Soc. Chem. Engrs, Japan, p. 700, Okayama (1978).

4) Harrison, D.: AIChE Symp. Ser., 69, No. 128, 14 (1973).

5) Hiby, J. W.: Chem-Ing-Tech., 36, 228 (1964).

6) Horio, M., C. Y. Wen, R. Krishnan, R. Khosravi and P. Rengarajan: Proceedings of the 2nd Pacific Chem. Eng. Congress, vol. II, p. 1182 (1977).

7) Ikeda, Y.: Kagaku Kögaku, 38, 632 (1974).

8) Kunii, D. and O. Levenspiel: "Fluidization Engineering", p. 87, John Wiley (1969).

9) Lefroy, G. A. and J. F. Davidson: Trans. Inst. Chem. Engrs., 47, 120 (1969).

10) Leung, L. S.: Powder Technology, 6, 189 (1972).

11) Lim, C. J. and K. B. Mathur: "Fluidization", ed. by J. F. Davidson and D. L. Keairns, p. 104, Cambridge Univ. Press (1978).

12) Makishima, S. and T. Shirai: J. Chem. Eng. Japan, 2, 124 (1969).

13) Makishima, S. and T. Shirai: Preprint of 34th Annual Meeting of The Soc. of Chem. Engrs., Japan, p. 176, Tokyo (1969).

14) Mamuro, T. and H. Hattori: J. Chem. Eng. Japan, 1, 1 (1968).

15) Merry, J. M. D.: AIChE J., 21, 507 (1975).

16) Mori, S. and A. Moriyama: Kagaku Kogaku Ronbunshu, 3, 7 (1977), [Int. Chem. Eng., 18, 245 (1978)].

17) Mori, S. and C.Y. Wen: "Fluidization Technology", ed. by D. L. Keairns, vol. 1, p. 179, Hemisphere Publishing Co. (1976).

18) Richardson, D. R.: Chem. Eng., 68, 83 (1961).

19) Siegel, R.: AIChE J., 22, 590 (1976).

20) Shinohara, H., I. Tanaka, S. Hiromasa and Y. Yoshimura: Preprint of the 9th Autumn Meeting of The Soc. of Chem. Engrs., Japan, p. 315, Fukuoka, (1975).

21) Toei, R., R. Matsuno, M. Oichi, K. Kubo and T. Yanagida: Kagaku Kogaku Ronbunshu, 1, 571 (1975).

22) Wen, C. Y., R. Krishnan, R. Khosravi and S. Dutta: "Fluidization", ed. by J. F. Davidson and D. L. Keairns, p. 32, Cambridge Univ. Press (1978).

23) Yoshimura, Y., T. Yamamoto, S. Hiromasa, I. Tanaka and H. Shinohara: Preprint of the 42nd Annual Meeting of The Soc. of Chem. Engrs. Japan, p. 483, Hiroshima (1977).

24) Zenz, F. A.: "Tripartite Chem. Eng. Conf., Symp. of Fluidization II". p. 36, Inst. Chem. Engrs. (1968). 